\title{
Faint extended structures near galaxies: preliminary results from the Wise Observatory
}

\author{
Noah Brosch ${ }^{1}$, Aleksandr Mosenkov ${ }^{2}$ and R. Michael Rich ${ }^{3}$ \\ ${ }^{1}$ Wise Observatory, Tel Aviv University and Dept. of Physics and Astronomy, Tel Aviv \\ University, Tel Aviv 69978, Israel \\ email: noah@wise.tau.ac.il \\ ${ }^{2}$ Ghent University, Belgium; St. Petersburg State University and Central Astronomical \\ Observatory, Russian Academy of Sciences, Russia ${ }^{3}$ Dept. of Physics and Astronomy, UCLA
}

\begin{abstract}
We present the first results from a survey of deep imaging of edge-on galaxies, with the goal of testing the growth-by-accretion of galaxies proposed by $\Lambda \mathrm{CDM}$. The data were obtained with a new telescope at the Wise Observatory. Our images show previously unreported extensions of the disk, tidal distortions, and streams at the level of 27-28 mag $\operatorname{arcsec}^{-2}$.
\end{abstract}

Keywords. Galaxies, halos, interactions

In $\Lambda$ CDM galaxies form by accreting dwarf-galaxy sized units of dark and baryonic matter. It is important to confront this prediction with observations; among the most powerful approach is the observation of the accretion history of galaxies. We have initiated a program of deep imaging for a sample of 180 edge-on disk galaxies (EODGs) selected from the Revised Catalog of Edge-on Galaxies (Karachentsev et al. 2003) with angular size $>2$ arcmin and visibility $\left(\delta>-30^{\circ}\right)$. We use a new $0.7-\mathrm{m}$ prime-focus telescope at the Wise Observatory (The Jay Baum Rich telescope; JBRT, Brosch et al. 2015), as well as an identical facility near Frazier Park, CA (see Rich et al. these proceedings). The JBRT camera is at prime focus behind a 2-element Ross corrector, imaging a $\sim 1 \square^{\circ}$ field at f/3.2 onto a PL-16801 (FLI) $4096^{2}$ CCD with 9 um pixels, at 0.84 arcsec pixel ${ }^{-1}$. To reach the required LSB levels we co-add $20-100+$ dithered images, each exposed for $300 \mathrm{sec}$ through a luminance (wide R) filter [250 nm bandpass, center at $560 \mathrm{~nm}$, flat transmission profile at $95 \%$ over the entire bandpass].

The images are debiased, dark-subtracted, flat-fielded with twilight sky flats, astrometrically registered, sky-subtracted and co-added, while rejecting outlier pixels, using THELI (Schirmer 2013). Further processing is done using DECA (Mosenkov 2014). This includes masking of unrelated objects (stars, field galaxies, artifacts), re-estimation of the sky background in the region of interest, and photometric calibration using unsaturated stars with R-band magnitudes. The galaxy image is then cropped and aligned with the major axis horizontal, and a model fit to the galaxy's surface brightness profile. Our preliminary very deep images of EODGs reach $\mu \geqslant 28$ mag $\operatorname{arcsec}^{-2}$. Imaging the entire EODG sample will be used to test $\Lambda$ CDM.

\section{References}

Brosch, N., Kaspi, S., Niv, S. \& Manulis, I. 2015 Ap\&SSS, 359, 49

Karachentsev, I. D., et al. 2003 arXiv:astro-ph/0305566

Mosenkov, A. 2014 Astrophys. Bull. 69 [1], 99

Schirmer, M. 2013 ApJS 209, 21 